\title{
Vertical Decomposition Approach to Solve Single Stage Capacitated Warehouse Location Problem (SSCWLP)
}

\author{
Priyanka Verma, Renduchintala Raghavendra Kumar Sharma \\ Department of Industrial and Management Engineering, Indian Institute of Technology, \\ Kanpur, India \\ E-mail: rrks@iitk.ac.in
}

Received May 4, 2011; revised May 25, 2011; accepted June 20, 2011

\begin{abstract}
Single Stage Capacitated Warehouse Location Problem (SSCWLP) has been attempted by few researchers in the past. These are Geoffrion and Graves [1], Sharma [2], Sharma [3] and Sharma and Berry [4]. In this paper we give a "vertical decomposition" approach to solve SSCWLP that uses Lagrangian relaxation. This way SSCWLP is broken into two versions of capacitated plant location problem (the CPLP_L and CPLP_R) by relaxing the flow balance constraints. For CPLP_R, we use well known Lagrangian relaxations given in literature (Christofides and Beasley [5] and Nauss [6]); and adopt them suitably for solving CPLP_L. We show theoretically in this paper that SSCWLP can be more efficiently solved by techniques of vertical decomposition developed in this paper than the method available in literature (Sharma and Berry [4]). Encouraging computational study is reported in this paper.
\end{abstract}

Keywords: Single Stage Capacitated Warehouse Location Problem, Linear Programming Relaxation, Lagrangian Relaxation, Vertical Decomposition

\section{Introduction and Literature Review}

The problem considered in this work is referred to as the single stage capacitated warehouse location problem (SSCWLP), which arises when the distances between plants and markets are large and it becomes necessary to route the supplies through warehouses (of limited capacities). A set of points are given where warehouses of limited capacities can be located. Each of these points has a known fixed cost associated with them. We are to choose a sufficient number of points where warehouses can be located so that sum total of location - distribution cost is minimized.

The study on the SSCWLP is motivated by its application in many fields, especially in supply chains in which hierarchical structure exists. Melo et al. [7] has reviewed different variants of multistage facility location problem and classified the types of problems attempted in this field on the basis of number of layers, number of location layers, number of commodities, nature of the planning horizon (single/multi period) and the type of data (deterministic/stochastic).
There are authors who have attempted for SSCWLP and its variants, some of these are discussed here. Geoffrion and Graves [1] gave a Bender's decomposition approach to solve multi-commodity SSCWLP. Later Hindi and Basta [8] address similar type of distribution design problem with consideration of operating cost per unit of commodity at warehouse along with the fixed cost associated with it. They used a branch and bound (B\&B) algorithm based on weak Linear Programming (LP) relaxation of the problem. Sharma [3] have attempted for a food distribution problem faced by Food Corporation of India (FCI), which is closer to multistage uncapacitated warehouse location problem. Later a solution procedure based on Lagrangian relaxation was developed for this. Klose [9] attempts for capacitated two-stage facility location problem with single source constraints. The developed model is a single commodity version of distribution design given by Geoffrion and Graves [1]. They give LP formulation, which is iteratively refined using valid inequalities and facets, and feasible solutions are obtained using simple heuristics by the information from solving LP. In this way good lower and upper bounds are 
developed. Tragantalerngsak et al. [10] addresses for two layer facility location problem, where second layer facility has limited capacity and service of customers is done by only one facility in the second layer. Simultaneously, second layer facility can be supplied by only one facility in the first layer. A Lagrangian relaxation (LR) based $\mathrm{B} \& \mathrm{~B}$ algorithm is given which is compared with LP based $B \& B$ and is shown to be efficient as it provide smaller sized tree and requires less CPU time. A realistic variant to the problem that measure customer satisfaction is attempted by Eskigun et al. [11] where an outbound supply chain network is designed considering operational dynamics of lead times, etc. They provide a Lagrangian based heuristic, which gives good quality solutions. Melachrinoudis et al. [12] uses physical programming approach to reconfigure a warehouse network through consolidation and elimination. Their model enables a decision maker to consider multiple criteria (i.e., cost, customer service, etc) and to express criteria preferences not in a traditional form of weights, but in ranges of different degrees of desirability. Farahani and Asgari [13] investigate locating some warehouses in a real-world military logistics system. They use multiple objective decision making techniques to determine the locations of warehouses. To assign each supported center to only one of the located warehouses, a set partitioning model is used. Melachrinoudis and Min [14] develop a mixedinteger programming model to solve the warehouse redesign problem to phase-out underutilized warehouses without deteriorating customer services. They validate the model by applying it to a real-world problem and by its sensitivity analyses. Keskin and Üster [15] attempted for SSCWLP and gave a scatter search-based heuristic approach for the problem.

It is to be noted that Geoffrion and Graves [1] and Sharma [2] have attempted the warehouse location problem very interestingly; their formulations are completely different from each other. Formulation given by Sharma [2] has reduced number of variables. Sharma and Berry [4] discuss in detail the differences in the approach to formulation of SSCWLP due to Geoffrion and Graves [1] and Sharma [2]. It is to be noted that the primal problem in the Bender's decomposition used by Geoffrion and Graves [1] and Sharma [2] has a min-cost-flow problem involving flow from plants to warehouse to markets. In this paper, we present a method of "Vertical Decomposition" that breaks the original problem into two problems. One involving flow from plants to warehouse (CPLP_L), two involving flow from warehouse to markets (CPLP_R). Using this approach we use the theory developed for capacitated plant location problem (CPLP) (Cornuejols, Sridharan, \& Thizy [16]) (by suitably extending it to adopt to new version of CPLP (referred to as CPLP_L)) to solve the original SSCWLP by using a Lagrangian relaxation. We finally show that this results in a better approach to solve SSCWLP (than the one given by Sharma and Berry [4]).

In Section 2 we give the formulation and different relaxations of SSCWLP. In Section 3 vertical decomposition approach is discussed. Section 4 gives the results of empirical investigation used to verify the theorems of Section 3. In Section 5 we give the detailed procedure to determine lower bounds and feasible solution with the use of these relaxations and the usage of the bounds obtained in determination of exact solution to SSCWLP. Section 6 provides computational results along with its analysis. We finally provide our conclusion on this approach in Section 7.

\section{Formulation and Relaxations of SSCWLP}

Here we give a mathematical formulation of SSCWLP using the style of Sharma and Sharma (2000). In later section, different relaxations of SSCWLP are developed and discussed.

\subsection{Index}

$i$ : $\quad$ Index for supply points (plants); $i=1, \cdots, I ; I=$ number of plants.

$j: \quad$ Index for warehouses; $j=1, \cdots, J ; J=$ number of potential warehouse points.

$k$ : $\quad$ Index for markets; $k=1, \cdots, K$; $K=$ number of markets.

\subsection{Definition of Constants}

$D_{k}: \quad$ Demand for the commodity at ' $k$ '

$d_{k}$ : Demand at market ' $\mathrm{k}$ ' as a fraction of the total

S : market demand; $\quad D_{k} / \sum_{k} D_{k}$

$\begin{array}{ll}S_{i}: & \text { Supply available at ' } i \text { ' } \\ s_{i} \text { : } & \text { Supply available at point ' } i \text { ' as a fraction of }\end{array}$

the total market demand; $S_{i} / \sum_{k} D_{k}$.

$f_{j}$ : $\quad$ Fixed cost of locating a warehouse at ' $j$ '

$C A P_{j}$ : Capacity of a warehouse at ' $j$ '

cap ${ }_{j}$ : Capacity of warehouse at ' $j$ ' , as a fraction of the total market demand; $C A P_{j} / \sum_{k} D_{k}$.

$c p w_{i j}$ : Cost of transporting $\sum_{k} D_{k}$ quantity of goods from ' $i$ ' to ' $j$ '.

$c w m_{j k}$ : Cost of transporting $\sum_{k} D_{k}$ quantity of goods from ' $j$ ' to ' $k$ '.

\subsection{Definition of Variables}

$X P W_{i j}$ : Quantity shipped from ' $i$ ' to ' $j$ '. $X W M_{j k}$ : Quantity shipped from ' $j$ ' to ' $k$ '. 
$x p w_{i j}$ : Quantity shipped from ' $i$ ' to ' $j$ ' as a fraction of total market demand; $x p w_{i j}=X P W_{i j} / \sum_{k} D_{k}$

$x w m_{j k}$ : Quantity shipped from ' $j$ ' to ' $k$ ' as a fraction of total market demand; $x w m_{j k}=X W M_{j k} / \sum_{k} D_{k}$.

$y_{j}$ : $\quad$ Location variable $(=1$ if warehouse is located at point ' $j$ ', 0 otherwise)

\subsection{Mathematical Formulation}

Minimize

$$
\begin{aligned}
& Z=\sum_{i} \sum_{j} c p w_{i j} x p w_{i j}+\sum_{j} \sum_{k} c w m_{j k} x w m_{j k}+\sum_{j} f_{j} y_{j} \\
& \text { Subject to: } \\
& \sum_{i} \sum_{j} x p w_{i j}=1 \\
& \sum_{j} \sum_{k} x w m_{j k}=1 \\
& x p w_{i j} \leq y_{j} s_{i} \quad \forall i, j \\
& x w m_{j k} \leq y_{j} d_{k} \forall j, k \\
& \sum_{i} x p w_{i j} \leq y_{j} c a p_{j} \quad \forall j \\
& \sum_{k} x w m_{j k} \leq y_{j} c a p_{j} \quad \forall j \\
& \sum_{j} x p w_{i j} \leq s_{i} \quad \forall i \\
& \sum_{j} x w m_{j k}=d_{k} \quad \forall k \\
& x p w_{i j} \geq 0 \quad \forall i, j \\
& x w m_{j k} \geq 0 \quad \forall j, k \\
& y_{j} \in(0,1) \quad \forall j \\
& \sum_{j} \operatorname{cap}_{j} y_{j} \geq 1 \\
& \sum_{i} x p w_{i j}=\sum_{k} x w m_{j k} \quad \forall j
\end{aligned}
$$

Just to emphasize that in the above formulation instead of using single letter names of variable and constant, we use multiple letter names (viz. $x p w, x w m, c p w, c w m$, etc) so that one can easily recall their meanings, that is, flow/cost between plant to warehouse (pw) or warehouse to market (wm) etc, while reading the paper.

First part of the objective function denotes the total cost of transporting the commodity from supply points to the warehouses. The second part is the transportation cost from warehouses to markets; and the last part is the fixed cost of locating warehouses. Constraint (2) and (5) are the supply constraint which ensures that the total commodity shipped out from a supply point to all warehouses can at most be equal to the total supply from that supply point. Constraint (2a) and (5a) ensures the total quantity received at any market to be equal to the demand at that market. Constraint (3) and (3a) link 0-1 integer variables $\left(y_{j}\right)$ and other distribution variables that are real and greater than zero. These ensure that if a warehouse is located at any point then quantities shipped in or out of that warehouse point are positive, else the quantities shipped will be zero, as the warehouse is not located. Constraint (4) and (4a) ensures that total quantity flown in and out of the warehouse does not exceed its capacity if it's located. We assume $\sum_{i} s_{j}>=1$ to ensure feasibility of the problem.

Non-negativity constraint on $x p w_{i j}$ and $x w m_{j k}$ are given by (7) and (7a); while (8) is the 0 -1 integer constraint on $y_{j}$. (11) is the flow balance constraint which ensures that total incoming quantity at a particular warehouse from all plants is equal to total outgoing quantity from the same warehouse to all the markets. (9) is a surrogate constraint. With so many constraints available, we can formulate SSCWLP in a variety of different ways. In these if (8) is replaced by (12) we obtain various LP relaxations. In addition, various relaxations can be obtained as Lagrangian relaxation (LR).

\subsection{Relaxations of SSCWLP}

We now develop different LP relaxations and LRs of SSCWLP formulated above. The notation $\mathrm{R}^{*}$ _O represent different relaxations of SSCWLP, where $(*)$ is a replacement for numbers 1 to 4 .

When integrality restrictions on $y_{j}$ are relaxed we obtain (10)

$$
0 \leq y_{i} \leq 1 \text { for all } j
$$

R1_O: (1); Subject to: (2)-(6), (2a)-(6a), (9) and (10).

Result 1: R1_O is equal to strong LP relaxation of SSCWLP given by Sharma and Berry (2007). Here restriction on $y_{j}$ is relaxed by (10).

Proof: It is easy to see.

R2_O: In this LR a constraint introducing upper $\left(P_{U}\right)$ and lower $\left(P_{L}\right)$ limits on the number of open plants is added up. i.e.

$$
P_{L} \leq \sum_{j=1}^{n} y_{j} \leq P_{U}
$$

This LR is obtained by dualizing (2), (2a), (5) and (5a). Let $\lambda x p w_{0}, \lambda x w m_{0}$ be the Lagrangian multipliers associated with (2), (2a) and $\lambda x p w_{i}, \lambda x w m_{k}$ with (5) and (5a) respectively. 


$$
\begin{aligned}
Z_{R 2_{-} O}= & \max _{\lambda x p w_{0}, \lambda x w m_{0}, \lambda x p w, \lambda x w m}\left[\min _{x p w, x w m, y} \sum_{i} \sum_{j}\left(c p w_{i j}+\lambda x p w_{0}+\lambda x p w_{i}\right) x p w_{i j}+\sum_{j} \sum_{k}\left(c w m_{j k}+\lambda x w m_{0}+\lambda x w m_{k}\right) x w m_{j k}\right. \\
& \left.+\sum_{j} f_{j} y_{j}-\sum_{i} \lambda x p w_{i} s_{i}-\sum_{k} \lambda x w m_{k} d_{k}-\lambda x p w_{0}-\lambda x w m_{0}\right]
\end{aligned}
$$

Hence objective (12); Subject to: (3), (3a), (4), (4a), (6), (6a), (7), (9) and (11).

R3_O: This is a LR obtained by dualizing (2), (2a), (6) and (6a). Hence objective is (14); Subject to: (3), (3a), (4), (4a), (6), (6a), (7), (8), (9).

R4_O: (1); Subject to: (2)-(6), (2a)-(6a), $(7,8)$ and (9).

The original problem SSCWLP is referred for comparison.

\section{Vertical Decomposition Approach for SSCWLP}

SSCWLP is a well known NP hard problem. Hence, in order to solve large sized and realistic instances of SSCWLP, we devise a decomposition procedure-called "vertical decomposition approach", which is described below.

The objective function (1) may be rewritten as:

Minimize $Z=f_{1}+f_{2}$, where

$$
\begin{aligned}
& f_{1}\left(x p w_{i j}, c p w_{i j}, y_{j}, f_{j}\right) \\
& =\min \sum_{i} \sum_{j} c p w_{i j} x p w_{i j}+\frac{1}{2} \sum_{j} f_{j} y_{j} \\
& f_{2}\left(x w m_{j k}, c w m_{j k}, y_{j}, f_{j}\right) \\
& \quad=\min \sum_{j} \sum_{k} c w m_{j k} x w m_{j k}+\frac{1}{2} \sum_{j} f_{j} y_{j}
\end{aligned}
$$

This restructured objective function becomes a motivation to introduce the vertical decomposition approach for solving SSCWLP. If we give a close look to the formulation of SSCWLP, it can be observed that (9) is the only constraint which involves both the flow variables $x p w$ and $x w m$. So, if (9) is relaxed, the problem decomposes into two versions of CPLP, each with one part $\left(f_{1}\right.$ or $f_{2}$ ) of the objective function shown above. We name this approach as vertical decomposition approach, because the full SSCWLP is decomposed into two parts left and right. The left part, CPLP_L, contains the variables and parameters of plants and warehouse only ( $x p w$ and y). Similarly, the right part, CPLP_R, contains the variables and parameters of warehouse and markets only ( $x w m$ and $y$ ). Therefore, in a sense, by vertical decomposition approach, the stages of the problem are decomposed to get smaller sized problems, which are relatively easier to solve.

Now, (9) has to be relaxed in order to make the
SSCWLP formulation amenable to vertical decomposition approach. We use $\lambda_{-}$flow $_{j}$ as the Lagrangian multipliers to dualize (11). The resulting formulation of CPLP_L, CPLP_R and SSCWLP, with (9) relaxed, are as shown below:

\section{CPLP_L:}

$$
\begin{aligned}
Z_{C P L P_{-} L}= & \min _{x p w, y} \sum_{i} \sum_{j}\left(c p w_{i j}+\lambda_{-} \text {flow }_{j}\right) x p w_{i j} \\
& +\frac{1}{2} \sum_{j} f_{j} y_{j}
\end{aligned}
$$

Subject to: (2), (3), (4), (5), (6), (7), (8) and (9). CPLP_R:

$$
\begin{aligned}
Z_{C P L P_{-} R}= & \min _{x w m, y} \sum_{j} \sum_{k}\left(c w m_{j k}-\lambda_{-} \text {flow }_{j}\right) x w m_{j k} \\
& +\frac{1}{2} \sum_{j} f_{j} y_{j}
\end{aligned}
$$

Subject to: (2a), (3a), (4a), (5a), (6a), (7), and (8).

\section{SSCWLP:}

$$
\begin{aligned}
Z_{S S C W L P}= & \max _{\lambda_{-} \text {flow }}\left[\min _{x p w, x w m, y} \sum_{i} \sum_{j}\left(c p w_{i j}+\lambda_{-} \text {flow } w_{j}\right) x p w_{i j}\right. \\
& \left.+\sum_{j} \sum_{k}\left(c w m_{j k}-\lambda_{-} \text {flow }_{j}\right) x w m_{j k}+\sum_{j} f_{j} y_{j}\right] \\
\text { or, } & \\
& Z_{S S C W L P}=\max _{\lambda_{-} \text {flow }}\left(Z_{C P L P_{-} L}+Z_{C P L P_{-} R}\right)
\end{aligned}
$$

Subject to: (2) to (9) and (2a) to (6a).

Here the problem SSCWLP is broken into sub problems CPLP_L and CPLP_R. This is vertical decomposition. Sharma [2] decomposed a multi commodity and multi period problem into single commodity and single period problem representing flow from plants to warehouses to markets. This is referred to as horizontal decomposition to bring out the contrast. In the next sub-section different relaxations of CPLP_L and CPLP_R are discussed in detail. Further, procedure to solve SSCWLP using these decomposed problems is discussed. Later a relationship theorem indicating the strength of different relaxations of SSCWLP is given.

\subsection{Relaxations of CPLP_L}

R1_L: (1a); Subject to: (2)-(6) and (10).

R2_L: In this LR is obtained by dualizing (2) and (6). 


$$
Z_{R 2_{-} L}=\max _{\lambda x p w_{0}, \lambda x p w x p w, y} \min _{i} \sum_{j}\left(c p w_{i j}+\lambda_{-} f l o w_{j}+\lambda x p w_{0}+\lambda x p w_{i}\right) x p w_{i j}+\frac{1}{2} \sum_{j} f_{j} y_{j}-\sum_{i} \lambda x p w_{i} s_{i}-\lambda x p w_{0}
$$

Subject to: (3), (4), (6), (7) and (11).

R3_L: (13); Subject to: (3), (4), (6)-(9).

R4_L: (1a); Subject to: (2), (3), (4), (5), (6), (7) and (8).

The main problem i.e. $\mathrm{Z}_{\mathrm{CPLP} \_\mathrm{L}}$ is referred as $\mathrm{R} 4 \_\mathrm{L}$ for comparison.

\subsection{Relaxations of CPLP_R}

R1_R: (1b); Subject to: (2a)-(6a) and (10). R1_R is a strong LP relaxation (follows from Davis and Ray (1969)).

R2_R: LR proposed by Christofides and Beasley [5]. This LR is obtained by dualizing (2a) and (6a).

$$
Z_{R 2_{-} R}=\max _{\lambda x w m_{0}, \lambda x w m_{k}} \min _{x w m, y} \sum_{j} \sum_{k}\left(c w m_{j k}-\lambda-f l o w_{j}+\lambda x w m_{0}+\lambda x w m_{k}\right) x w m_{j k}+\frac{1}{2} \sum_{j} f_{j} y_{j}-\sum_{k} \lambda w m_{k} d_{k}-\lambda x w m_{0}
$$

Subject to: (3a), (4a), (6a), (7), (1).

R3_R: (14); Subject to: (3a), (4a), (6a), (7), (8). It is attempted by Nauss [6].

R4_R: (1b); Subject to: (2a), (3a), (4a), (5a), (6a), (7), and (8).

The main problem i.e. $\mathrm{Z}_{\mathrm{CPLP} \_\mathrm{R}}$ is referred as $\mathrm{R} 4 \_\mathrm{R}$ for comparison.

\subsection{Relationship between Relaxations of CPLP_L}

Note that CPLP_L is different from CPLP_R. In this section, a comparison of the strength of the bounds given by different relaxations of CPLP_L is given. We constructed the proofs and found that they are marginally different from CPLP_R (Cornuejols, Sridharan, \& Thizy [16].

Theorem 1: $Z_{R 1_{-} L} \leq Z_{R 2_{-} L} \leq Z_{R 3_{-} L} \leq Z_{R 4_{-} L}$.

This theorem provides the relative effectiveness of the bounds that may be obtained for these relaxations of CPLP_L.

\subsection{Relationship between Relaxations of CPLP_R}

Cornuejols, Sridharan, \& Thizy [16] have given relative strength of different relaxations of standard CPLP which is similar to CPLP_R. Hence, the same relaxations along with their relative strength are used for CPLP_R here in form of theorem 2 below.

Theorem 2: $Z_{R 1_{-} R} \leq Z_{R 2_{-} R} \leq Z_{R 3_{-} R} \leq Z_{R 4_{-} R}$.

This theorem provides the relative effectiveness of the bounds that may be obtain for these relaxations of CPLP_R.

\subsection{Relationship between Relaxations of SSCWLP}

Here a comparison of the strength of the bounds, given by different relaxations of SSCWLP based on CPLP_L and CPLP_R is given.

Proposition 1: The bounds obtained by relaxations R1_O and R2_O are related as $Z_{R 1 \_o} \leq Z_{R 2 \_o}$.

Proof: With the flow balance constraint (9) relaxed, objective of R2_O can be written as:

$$
\begin{aligned}
Z_{R 2_{-} O}= & \max _{\substack{\lambda x p w_{0}, \lambda x p w, \lambda x w m_{0}, \lambda x w m, \lambda_{-} f l o w}} \min _{x p w, x w m, y} \sum_{i} \sum_{j}\left(c p w_{i j}+\lambda_{-} f l o w_{j}+\lambda x p w_{0}+\lambda x p w_{i}\right) x p w_{i j} \\
& +\sum_{j} \sum_{k}\left(c w m_{j k}-\lambda_{-} f l o w_{j}+\lambda x w m_{0}+\lambda x w m_{k}\right) x w m_{j k}+\sum_{j} f_{j} y_{j}-\sum_{i} \lambda x p w_{i} s_{i}-\sum_{k} \lambda x w m_{k} d_{k}-\lambda x p w_{0}-\lambda x w m_{0}
\end{aligned}
$$

Subject to: (4), (4a), (6), (6a), (7).

$$
\begin{aligned}
Z_{R_{2} O}= & \left\{\max _{\lambda_{-} f l o w_{j}} \max _{\substack{\lambda x p w_{0}, \lambda x p w, x p w, x w m, y \\
\lambda x w m_{0}, \lambda x w m}} \sum_{i} \sum_{j}\left(c p w_{i j}+\lambda \text { flow }_{j}+\lambda x p w_{0}+\lambda x p w_{i}\right) x p w_{i j}\right. \\
& \left.+\sum_{j} \sum_{k}\left(c w m_{j k}-\lambda_{-} \text {flow }_{j}+\lambda x w m_{0}+\lambda x w m_{k}\right) x w m_{j k}+\sum_{j} f_{j} y_{j}-\sum_{i} \lambda x p w_{i} s_{i}-\sum_{k} \lambda x w m_{k} d_{k}-\lambda x p w_{0}-\lambda x w m_{0}\right\}
\end{aligned}
$$

Subject to: (4), (4a), (6), (6a), (7).

$$
Z_{R_{2} O}=\max _{\lambda_{-} \text {flow }_{j}}\left\{Z_{R 2_{-} L}+Z_{R_{-} R}\right\}
$$

According to the proofs earlier shown, $Z_{R_{1} R} \leq Z_{R_{2}{ }_{-} R}$ [for CPLP_R] and $Z_{R 1_{-} L} \leq Z_{R 2_{-} L}$ [for CPLP_L].

Hence, $Z_{R 2_{-} O} \leq \max _{\lambda \_ \text {flow }}\left\{Z_{R 1_{-} L}+Z_{R 1_{-} R}\right\}$. 
Note that here (9) is excluded. If (9) is also included, then the problem become R1_O subjected to its usual constraints. i.e. $Z_{R 1_{-} O} \leq Z_{R 2_{-} O}$.

Proposition 2: $Z_{R 2_{-} O} \leq Z_{R 3_{-} O}$.

Proof: We find that all the results of original problem have identical proof. Hence proof for Proposition 1 has been shown and the proofs for Proposition 2 being similar to it are omitted. When these propositions are combined, following theorem is developed showing relative strength of bounds given by different relaxations of SSCWLP.

Theorem 3: $Z_{R 1 \_0} \leq Z_{R 2 \_0} \leq Z_{R 3 \_0} \leq Z_{R 4 \_0}$.

Strongest LP relaxation of SSCWLP proposed by Sharma and Berry [4] is same as relaxation R1_O of SSCWLP proposed. From theorem 3, we observe that the lagrangian relaxations R2_O and R3_O can be better than the strongest relaxations known of SSCWLP (that is R1_O). Empirical study given below shows that differences in performances of relaxations considered in theorem 3 are statistically significant.

\section{Empirical Investigation for CPLP_L and CPLP_R}

Here we give results of an empirical study. We find that there is significant difference in the performance of different relaxations considered in theorem 3.

Sample problems for SSCWLP sized $50 \times 50 \times 50$ were randomly created using C codes; a $50 \times 50 \times 50$ problem corresponds to a set of 50 plants, 50 warehouses and 50 markets. Two categories of problems are considered-nil category (A) and abundance category (B); the same is tabulated in Table 1 below.

For each of these categories, we created 25 problems in which fixed location cost and the transportation cost are uniformly distributed as shown in Table 1. For each problem instance, we obtained the value of different relaxations of CPLP_R and CPLP_L using GAMS 22.3 in a Pentium D $2.80 \mathrm{GHz}, 1$ GB RAM computer. Sub-gradient optimization is used for solving Lagrangian relaxations of CPLP_L and CPLP_R. Lagrangian relaxation method is powerful in the sense that it gives good lower bounds (for the minimization problem) in competitive computational time. Starting Lagrangian multiplier is taken either to be 0 or maximum value of $\left(0.5^{*}\right.$ cap $\left._{j} / f_{j}\right)$ from all $j$ warehouses. Using trial and error approach between these two choices, good results were obtained. Details can be seen in appendix A. Here we take percentage improvement between a pair of relaxations (to generate normalized data) for every problem instance; and then t-test is performed.

t-tests for bounds of the different relaxations for different categories are shown in Tables 2 and 3. These t-values are compared with those in Table 4.

Note that each cell of the above table shows the t-value of the t-test between the two relaxations represented by the respective row/column of the matrix. For e.g. t-value between R1 and R2 shows the t-test done between 0 and $100 *(\mathrm{R} 2-\mathrm{R} 1) / \mathrm{R} 1$. Table 2 shows the t-values obtained for differences in bounds of relaxations for category A problems; and Table $\mathbf{3}$ is revealing the t-values for differences in bounds of relaxations for category B problems.

\subsection{Analysis for Category A Problems}

When comparing t values for bounds of different relaxations of CPLP_L [Table 2], the LP relaxation R1_L is giving significantly better bounds as compared to rest all relaxations. We observe from Table 2 that linear relaxation R1_R is the best performing relaxation for category A problems.

Table 1. Problem categories for SSCWLP.

\begin{tabular}{|c|c|c|c|c|}
\hline \multirow[b]{2}{*}{ Problem category } & \multicolumn{2}{|c|}{ Parameters } & \multirow[b]{2}{*}{ Fixed location cost } & \multirow[b]{2}{*}{ Transportation Cost } \\
\hline & $\sum_{i} s_{i}$ & $\sum_{j} c a p_{j}$ & & \\
\hline A & 1.0 & 1.0 & $\mathrm{U}[100,1000]$ & $\mathrm{U}[1,100]$ \\
\hline B & 5.0 & 5.0 & $\mathrm{U}[100-1000]$ & $\mathrm{U}[1,100]$ \\
\hline
\end{tabular}

Table 2. t-test for differences in bounds for category A problems.

\begin{tabular}{|c|c|c|c|c|c|c|c|c|c|}
\hline \multirow{2}{*}{ t-test } & \multicolumn{4}{|c|}{ CPLP_L } & \multirow{2}{*}{ t-test } & \multicolumn{4}{|c|}{ CPLP_R } \\
\hline & $\mathrm{R} 1$ & $\mathrm{R} 2$ & R3 & $\mathrm{R} 4$ & & $\mathrm{R} 1$ & $\mathrm{R} 2$ & R3 & $\mathrm{R} 4$ \\
\hline $\mathrm{R} 1$ & & $-23.1^{+}$ & $-23.1^{+}$ & -0.16 & R1 & & $-24.3^{+}$ & $-24.7^{+}$ & 1.44 \\
\hline $\mathrm{R} 2$ & & & 1 & $23.35^{+}$ & $\mathrm{R} 2$ & & & $2.2^{+++}$ & $24.3^{+}$ \\
\hline R3 & & & & $23.35^{+}$ & R3 & & & & $24.71^{+}$ \\
\hline
\end{tabular}


Table 3. t-test for differences in bounds for category B problems.

\begin{tabular}{|c|c|c|c|c|c|c|c|c|c|}
\hline \multirow{2}{*}{ t-test } & \multicolumn{4}{|c|}{ CPLP_L } & \multirow{2}{*}{ t-test } & \multicolumn{4}{|c|}{ CPLP_R } \\
\hline & $\mathrm{R} 1$ & R2 & R3 & R4 & & R1 & R2 & R3 & R4 \\
\hline R1 & & 1.01 & $6.29^{+}$ & 6.35 & R1 & & -0.61 & $6.29^{+}$ & $6.29^{+}$ \\
\hline R2 & & & $6.29^{+}$ & $6.35^{+}$ & R2 & & & $6.29^{+}$ & $6.29^{+}$ \\
\hline R3 & & & & 1.63 & R3 & & & & $3.18^{+}$ \\
\hline
\end{tabular}

Table 4. t-critical value (Table value).

\begin{tabular}{cccc}
\hline Significance level & $\alpha=0.05$ & $\alpha=0.01$ & $\alpha=0.005$ \\
\hline t-critical (one tail) & 1.68 & 2.40 & 2.68 \\
\hline
\end{tabular}

${ }^{++}:$At $\alpha=0.05$, significance level $=1$ tail; ${ }^{++}$: At $\alpha=0.01$, significance level $=1$ tail; ${ }^{+}$: At $\alpha=0.005$, significance level $=1$ tail.

\subsection{Analysis for Category B Problems}

When comparing t-values from Table 3 and Table 4, it is found that most of the relaxations are following theorem-1 and theorem-2 respectively for CPLP_L as well as CPLP_R. Significant t-values indicate that the LR R3_L of CPLP_L and R3_R of CPLP_R are providing significantly better bounds. This results in faster execution of the branch and bound procedure based procedure to solve SSCWLP as shown in the next section.

\section{Branch and Bound Procedure to Solve SSCWLP}

In this section we aim to use three best performing relaxations to determine bounds in a branch and bound procedure. It is known that stronger the relaxations, better the bounds, lesser will be the nodes traversed in an enumeration tree and hence one achieves computational advantage. In R2_O and R3_O we relax the flow balance constraints and solve the associated CPLP_R and CPLP_L by lagrangian relaxation procedure. Details of lagrangian relaxation procedure is given in Sharma (1991). The sketch of branch and bound procedure to solve SSCWLP is given below.

\subsection{Procedure Branch and Bound}

Step 1: Initialization. Set current_best_solution = INFINITY;

best_lower_bound = - INFINITY;

Node [1]. Fixed Var List $=\{\mathrm{y} 1=0\}$;

Node [1]. Free Var List $=\{2, \cdots, N\}$;

Node [2]. Fixed Var List $=\{\mathrm{y} 1=1\}$;

Node [2]. Free Var List $=\{2, \cdots, N\}$;

Add nodes 1 and 2 to list $\mathrm{A}$.

Step 2: If list A is empty
Then Stop. Optimal Solution Found

Else pick up top node (TN) from list A

Step 3: Compute lagrangian bound (associated with R2_O or R3_O) or LP relaxation bound (associated with R1_O) associated with node TN (referred to as TN.R_ Bound). If (TN_R_Bound > best_lower_bound), then best_lower_bound = TN_R_Bound.

Solve associated min cost flow problem (with TN) and generate a feasible solution for SSCWLP (referred as TN_feasible_solution). If (TN_feasible_solution < current_best_solution), then current_best_solution $=\mathrm{TN}_{-}$ feasible_solution.

If (TN.R_Bound > current_best_solution)

Then node TN gets fathomed. Go to step 2 .

Else If free variables associated with TN $>=1$

Then generate two more nodes by setting a chosen free variable associated with $\mathrm{TN}$ at 0 and 1; make relevant updates and add these nodes to list A.

Else No more branching possible; go to step 2.

The above procedure is repeated separately for R1_O, R2_O and R3_O to facilitate a comparison. Empirical investigation is given below.

\section{Empirical Investigation for SSCWLP}

In the earlier sections, the relationship theorem indicating the strength of different relaxations of CPLP_L, CPLP_R and SSCWLP is shown. Also solution procedures of some of the best performing relaxations of SSCWLP using vertical decomposition approach are discussed. In this section we focus on usage of these relaxations of SSCWLP in a branch and bound procedure to obtain an optimal solution. Performance of these relaxations with its application in a branch and bound is compared with a standard branch and bound procedure (BB) using strong LP relaxation (R1_O) of SSCWLP as its lower bound. We thus compare the new proposed relaxations with that of the best possible known relaxation, R1_O (Sharma and Berry, 2007), and show its efficacy by the reduction on nodes and time achieved. The objective of this section is to study how well these relaxations do relative to each other. In particular, we are interested in looking at the influence of supplies versus capacities, capacities versus demands, and fixed costs 
versus transportations costs on the bounds provided by these relaxations, and the solutions provided by the heuristics.

Sample problems for SSCWLP, sized $50 \times 50 \times 50$ and $100 \times 100 \times 100$ (plants $\times$ warehouse $\times$ markets) were created randomly. From the empirical investigations done for CPLP_L and CPLP_R, we have observed that relationship theorems 1 and 2 are satisfied for the abundance case more promptly. Hence for conducting empirical experiments for complete problem of SSCWLP we have considered varying categories of abundance in supply and capacity limits. Four categories of problems with varying problem size and supply - capacity limits were prepared details of which are given in Table 5 .

We solved 20 problem instances in each of the category of P1, P2, P3 and P4 on Pentium D 2.80 GHz, 1 GB RAM computer. All the algorithms are coded in MATLAB software with calls to GAMS22.3 for solving the mincost-flow problem. Details are given in appendix B. We compute percentage improvement for any two methods for every problem instance (to generate normalized data) and then t-tests are performed on the number of nodes and the time required to solve them; these are shown in Table 6.

For each Rm-Rn ("m” and " $n$ " are BB, 1, 2, 3 as shown in column 3 of Table 6), "Nodes" is " $t$ " calculated for the difference between (Number of nodes taken with formulation Rm/Number of nodes taken with formulation $\mathrm{Rn}$ ) and 1. Similarly, "Time" is "t" calculated for difference between CPU time for Rm/CPU time for Rn) and 1. BB refers to the complete enumeration for solving the problem. Nodes under BB refer to the number of nodes taken to solve the problem. Negative $t$ value indicates reduced number of node and reduced execution time.

\subsection{Analysis for Category P1 and P3 Problems}

Category P1 and P3 problems have got the same variation in supply and capacity limits, but their problem sizes are different. For the problem category P1, we note from the Table 6 that the performance (in terms of reduction in number of nodes) of relaxation R1_O is significantly better than BB. Similarly R2_O is significantly better performer as compared to R1_O; however performance of

Table 5. Problem categories for SSCWLP.

\begin{tabular}{cccccc}
\hline \multirow{2}{*}{ Problem category } & Problem Size & \multicolumn{2}{c}{ Parameters } & & \\
\cline { 3 - 4 } & & $\sum_{i} s_{i}$ & $\sum_{j} c a p_{j}$ & Fixed Location Cost & Transportation Cost \\
\hline P1 & $50 \times 50 \times 50$ & 2.5 & 2.5 & $\mathrm{U}[100,1000]$ & $\mathrm{U}[1,100]$ \\
P2 & $50 \times 50 \times 50$ & 10 & 10 & $\mathrm{U}[100,1000]$ & $\mathrm{U}[1,100]$ \\
P3 & $100 \times 100 \times 100$ & 2.5 & 2.5 & $\mathrm{U}[1500,2000]$ & $\mathrm{U}[1,100]$ \\
P4 & $100 \times 100 \times 100$ & 10 & 10 & $\mathrm{U}[1500,2000]$ & $\mathrm{U}[1,100]$ \\
\hline
\end{tabular}

Table 6. t-test for Nodes and time taken to solve the problems.

\begin{tabular}{|c|c|c|c|c|}
\hline Problem category & Problem Size & Comparison between ' $\mathrm{Rm}-\mathrm{Rn}$ ' & Nodes & Time \\
\hline \multirow{3}{*}{$\mathrm{P} 1$} & \multirow{3}{*}{$50 \times 50 \times 50$} & $\mathrm{R} 1 \_\mathrm{O}-\mathrm{BB}$ & $-5.02^{+}$ & $-5.24^{+}$ \\
\hline & & R2_O - R1_O & $-3.12^{+}$ & $-3.53^{+}$ \\
\hline & & R3_O - R2_O & $-2.75^{+}$ & $-1.76^{+}$ \\
\hline \multirow{3}{*}{$\mathrm{P} 2$} & \multirow{3}{*}{$50 \times 50 \times 50$} & R1_O - BB & $-2.67^{++}$ & $-2.92^{+}$ \\
\hline & & R2_O - R1_O & -1.32 & $-1.8^{+++}$ \\
\hline & & R3_O - R2_O & 1 & $-1.96^{+++}$ \\
\hline \multirow{3}{*}{ P3 } & \multirow{3}{*}{$100 \times 100 \times 100$} & R1_O - BB & $-5.07^{+}$ & $-5.1^{+}$ \\
\hline & & R2_O - R1_O & $-5.56^{+}$ & $-5.72^{+}$ \\
\hline & & R3_O - R2_O & $-3.82^{+}$ & $-3.83^{+}$ \\
\hline \multirow{3}{*}{$\mathrm{P} 4$} & \multirow{3}{*}{$100 \times 100 \times 100$} & R1_O - BB & $-2.22^{+++}$ & $-2.47^{++}$ \\
\hline & & R2_O - R1_O & $-3.83^{+}$ & $-3.82^{+}$ \\
\hline & & R3_O - R2_O & $-3.1^{+}$ & $-3.28^{+}$ \\
\hline
\end{tabular}


R3_O is still better than R2_O. Also higher t values indicate that superiority of R1_O over BB is most significant and R3_O over R2_O is least (however it is still significant). Exactly similar is the behavior of performance in terms of time taken to solve the problems. That is $\mathrm{R} 3$ _O is the best performer, superior to R2_O, which is better than R1_O; BB is the worst performer in terms of time.

Now when the problem size increases, that is for the problem category P3, it can be observed that R3_O still remains the best performer in terms of reduction in number of nodes as well as time taken to solve a problem. The relaxations R2_O and R1_O (in that sequence) follow R3_O; however BB still remains the worst performer, both in terms of nodes as well as solution time. Also as can be observed from the $t$ values, the superior performance of R2_O over R1_O is most significant; the pairs "R1_O over BB" and "R3_O over R2_O" follows in that sequence.

So it can be inferred from here that the use of relaxation R3_O is most suited to solve small as well as large sized SSCWLP, when there is a moderate level (2.5 times) of over-supply and over-capacity. Solving SSCWLP of category P1 and P3 using Branch and Bound is the least efficient method; however the use of R1_O, R2_O and R3_O (in ascending order of better performance) to determine bounds in a Branch and Bound method proves to be very fruitful.

\subsection{Analysis for Category P2 and P4 problems}

Category P2 and P4 problems have got the same variation in supply and capacity limits, but their problem size is different. It can be observed from the Table 6 that for problem category P2, relaxation R1_O performs significantly better, both in terms of reduction in number of nodes traversed as well as solution time, than the usual branch and bound method (BB). However, there is not any significant improvement in the performance of "R2_O over R1_O” or "R3_O over R2_O”.

However when the problem size increases, that is for problem category P4, R1_O performs better than BB, R2_O performs better than R1_O, and R3_O performs significantly better than R2_O both in terms of reduction in number of nodes as well as reduction in the time required to solve a problem. Also observing the $t$ values, it is evident that the significant performance of R1_O over BB is not as noteworthy as the significance of " $\mathrm{R} 2$ _O over R1_O” or "R3_O over R2_O”.

So it can be inferred that when level of over-supply and over-capacity is high (10 times), it is better to solve a smaller sized SSCWLP using R1_O (or R2_O or R3_O) and a large sized SSCWLP using R3_O. Also in this category for smaller sized problems, the use of R1_O, $\mathrm{R} 2$ _O or R3_O is equally better option compared to the branch and bound method because both R2_O and R3_O perform same as R1_O. However for large sized problems of this category, with the use of relaxations R1_O, R2_O and R3_O (in ascending order of better performance) as bounds in the branch and bound method perform better than the usual branch and bound technique.

We have shown that lagrangian based branch and bound procedures (R2_O and R3_O) are superior to R1_O (Sharma and Berry [4]) by implementing these algorithms on the common platform of MATLAB. It may be noted that BB and R1_O are solvable by commercially available packages as LINGO, CPLEX or GAMS; whereas lagrangian relaxation based procedures (R2_O and R3_O) are not directly solvable by these commercially available packages. First author of this paper (a Ph D candidate then) coded these algorithms on MATLAB; and there is enormous scope for improvement before its performance be compared to commercially available packages like CPLEX. However it is to be noted that Sharma and Berry [4] showed that R1_o is significantly superior to plain branch and bound procedure (BB) compared on commercially available LINGO software. Thus we provide preliminary evidence that R2_O and R3_O have significant merit for solving SSCWLP.

\section{Conclusions}

The contribution of this work in the existing vast literature of location problems is two folds. First is the introduction of "vertical decomposition" approach for SSCWLP, which can easily be extended to the multi stage warehouse location problems, or to the problems of different domains that are modeled in a manner similar to SSCWLP. Vertical decomposition approach allows us to decompose the complex and large sized SSCWLP into two versions of the standard CPLP. One of the decomposed problems, CPLP_R, is well researched and has different known relaxations. For the other problem, CPLP_L, we provide different relaxation and show that some of them are similar to CPLP_R. A relationship theorem of different relaxations shows the superiority of some relaxations over the others. In particular we show theoretically that for SSCWLP better Lagrangian relaxation exists than the LP relaxation given by Sharma and Berry [4]. Computational studies give support to our theoretical propositions.

Second and the major contribution of this work is to show the efficacy of "vertical decomposition" approach, by using the best performing relaxations of the decomposed and original SSCWLP to advantageously determine an exact solution to the large sized SSCWLP. 
Three best performing relaxations are selected based on their relative efficiencies given in relationship theorems, and a procedure to solve them is also provided. These relaxations are used to determine lower bound and a feasible solution, which in turn are used in a branch and bound method. Computational study is done for a variety of problems of different sizes. It is found that one of the Lagrangian relaxations (R3_O) is performing best in terms of time and nodes travelled in almost all the cases, as compared to the remaining relaxations. This is a significant finding as relaxation R1_O was found to be the best performer for SSCWLP by Sharma and Berry [4]; that is we actually landed up finding a relaxation of SSCWLP which is better than that existing in literature. A future research possibility with huge potential could be to extend the results of this paper to multistage location distribution problems, which can be modeled using the "vertical decomposition" approach.

\section{References}

[1] A. M. Geoffrion and G. W. Graves, "Multicommodity Distribution System Design by Benders Decomposition,” Management Science, Vol. 20, No. 5, 1974, pp. 822-844. doi:10.1287/mnsc.20.5.822

[2] R. R. K. Sharma, "Modeling a Fertilizer Distribution System," European Journal of Operational Research, Vol. 51, No. 1, 1991, pp. 24-34. doi:10.1016/0377-2217(91)90142-I

[3] R. R. K. Sharma, "Food Grains Distribution in the Indian Context: An Operational Study,” In: A. Tripathi and J. Rosenhead, Eds., Operations Research for Development, New Age International Publishers, New Delhi, 1996, pp. 212-227.

[4] R. R. K. Sharma and V. Berry, "Developing New Formulations and Relaxations of Single Stage Capacitated Warehouse Location Problem (SSCWLP): Empirical Investigation for Assessing Relative Strengths and Computational Effort," European Journal of Operational Research, Vol. 177, No. 2, 2007, pp. 803-812. doi:10.1016/j.ejor.2005.11.028

[5] N. Christofides and J. E. Beasley, "Extensions to a Lagrangean Relaxation Approach for the Capacitated Warehouse Location Problem," European Journal of Operational Research, Vol. 12, No. 1, 1983, pp. 19-28. doi:10.1016/0377-2217(83)90179-0

[6] R. M. Nauss, “An Improved Algorithm for the Capaci- tated Facility Location Problem,” Journal of Operational Research Society, Vol. 29, No. 12, 1978, pp. 1195-1201.

[7] M. T. Melo, S. Nickel and F. Saldanha-da-Gama, "Facility Location and Supplychain Management-A Review," European Journal of Operational Research, Vol. 196, No. 2, 2009, pp. 401-412. doi:10.1016/j.ejor.2008.05.007

[8] K. S. Hindi and T. Basta, "Computationally Efficient Solution of a Multiproduct, Two-Stage Distribution-Location Problem," The Journal of the Operational Research Society, Vol. 45, No. 11, 1994, pp. 1316-1323.

[9] A. Klose, "An LP-Based Heuristic for Two-Stage Capacitated Facility Location Problems," The Journal of the Operational Research Society, Vol. 50, No. 2, 1999, pp. 157-166.

[10] S. Tragantalerngsak, J. Holt and M. Rönnqvist, “An Exact Method for the Two-Echelon, Single-Source, Capacitated Facility Location Problem," European Journal of Operational Research, Vol. 123, No. 3, 2000, pp. 473-489. doi:10.1016/S0377-2217(99)00105-8

[11] E. Eskigun, R. Uzsoy, P. V. Preckel, G. Beaujon, S. Krishnan and J. D. Tew, "Outbound Supply Chain Network Design with Mode Selection, Lead Times and Capacitated Vehicle Distribution Centers," European Journal of Operational Research, Vol. 165, No. 1, 2005, pp. 182-206. doi:10.1016/j.ejor.2003.11.029

[12] E. Melachrinoudis and H. Min, "Redesigning a Warehouse Network," European Journal of Operational Research, Vol. 176, No. 1, 2007, pp. 210-229. doi:10.1016/j.ejor.2005.04.034

[13] R. Z. Farahani and N. Asgari, "Combination of MCDM and Covering Techniques in a Hierarchical Model for Facility Location: A Case Study," European Journal of Operational Research, Vol. 176, No. 3, 2007, pp. 1839-1858. doi:10.1016/j.ejor.2005.10.039

[14] E. Melachrinoudis, A. Messac and H. Min, "Consolidating a Warehouse Network: A Physical Programming Approach," International Journal of Production Economics, Vol. 97, No. 1, 2005, pp. 1-17. doi:10.1016/j.ijpe.2004.04.009

[15] B. B. Keskin and H. Üster, "A Scatter Search-Based Heuristic to Locate Capacitated Transshipment Points," Computers \& Operations Research, Vol. 34, No. 10, 2007, pp. 3112-3125. doi:10.1016/j.cor.2005.11.020

[16] G. Cornuejols, R. Sridharan and J. M. Thizy, “A Comparison of Heuristics and Relaxations for the Capacitated Plant Location Problem," European Journal of Operational Research, Vol. 50, No. 3, 1991, pp. 280-297. doi:10.1016/0377-2217(91)90261-S 


\section{Appendix A}

Computational results for LHSCPLP, category A problem $50 \times 50 \times 50$.

\begin{tabular}{|c|c|c|c|c|c|c|c|c|}
\hline \multirow{2}{*}{ S.No } & \multicolumn{2}{|c|}{ R1_LHS } & \multicolumn{2}{|c|}{ R2_LHS } & \multicolumn{2}{|c|}{ R3_LHS } & \multicolumn{2}{|c|}{ R4_LHS } \\
\hline & Objective & Iteration & Objective & Iteration & Objective & Iteration & Objective & Iteration \\
\hline 1 & 15171.58 & 2227.00 & 15169.882 & 34 & 15169.885 & 37 & 15171.59 & 59 \\
\hline 2 & 13103.62 & 1992.00 & 13102.209 & 35 & 13102.21 & 35 & 13103.62 & 81 \\
\hline 3 & 13395.97 & 2158.00 & 13393.873 & 37 & 13393.874 & 37 & 13395.96 & 65 \\
\hline 4 & 13141.67 & 1985.00 & 13139.668 & 37 & 13139.672 & 37 & 13141.67 & 78 \\
\hline 5 & 12755.00 & 2030.00 & 12753.723 & 35 & 12753.727 & 35 & 12755 & 58 \\
\hline 6 & 13424.41 & 2957.00 & 13422.896 & 35 & 13422.896 & 35 & 13424.41 & 67 \\
\hline 7 & 11782.85 & 2320.00 & 11781.386 & 35 & 11781.386 & 35 & 11782.85 & 64 \\
\hline 8 & 15200.70 & 2319.00 & 15198.726 & 37 & 15198.727 & 37 & 15200.7 & 76 \\
\hline 9 & 12873.67 & 2529.00 & 12872.091 & 35 & 12872.094 & 35 & 12873.67 & 82 \\
\hline 10 & 13231.09 & 2303.00 & 13229.122 & 37 & 13229.123 & 37 & 13231.09 & 59 \\
\hline 11 & 11347.41 & 2638.00 & 11345.71 & 35 & 11345.71 & 35 & 11347.41 & 75 \\
\hline 12 & 13626.14 & 2633.00 & 13624.432 & 37 & 13624.432 & 35 & 13626.14 & 79 \\
\hline 13 & 12858.80 & 2350.00 & 12857.204 & 35 & 12857.204 & 35 & 12858.8 & 75 \\
\hline 14 & 13592.69 & 2548.00 & 13591.355 & 35 & 13591.355 & 35 & 13592.69 & 41 \\
\hline 15 & 13970.07 & 2257.00 & 13969.255 & 35 & 13969.255 & 35 & 13970.07 & 40 \\
\hline 16 & 13348.74 & 2591.00 & 13347.718 & 35 & 13347.722 & 35 & 13348.74 & 87 \\
\hline 17 & 13164.99 & 3069.00 & 13163.302 & 37 & 13163.314 & 35 & 13164.99 & 82 \\
\hline 18 & 14130.20 & 2458.00 & 14128.861 & 35 & 14128.866 & 35 & 14130.2 & 88 \\
\hline 19 & 13469.87 & 2459.00 & 13468.141 & 37 & 13468.141 & 37 & 13469.87 & 71 \\
\hline 20 & 14493.84 & 2414.00 & 14491.808 & 37 & 14491.808 & 37 & 14493.84 & 65 \\
\hline 21 & 13842.17 & 2105.00 & 13840.31 & 37 & 13840.31 & 37 & 13842.17 & 82 \\
\hline 22 & 13700.39 & 1903.00 & 13698.911 & 35 & 13698.911 & 35 & 13700.39 & 81 \\
\hline 23 & 14183.09 & 2225.00 & 14181.863 & 35 & 14181.863 & 35 & 14183.09 & 74 \\
\hline 24 & 13956.53 & 2320.00 & 13955.301 & 35 & 13955.301 & 35 & 13956.53 & 77 \\
\hline 25 & 14220.34 & 2192.00 & 14219.027 & 35 & 14219.027 & 35 & 14220.34 & 81 \\
\hline
\end{tabular}


Computational results for RHSCPLP, category A problem $50 \times 50 \times 50$.

\begin{tabular}{|c|c|c|c|c|c|c|c|c|}
\hline \multirow{2}{*}{ S.No } & \multicolumn{2}{|c|}{ R1_RHS } & \multicolumn{2}{|c|}{ R2_RHS } & \multicolumn{2}{|c|}{ R3_RHS } & \multicolumn{2}{|c|}{ R4_RHS } \\
\hline & Objective & Iteration & Objective & Iteration & Objective & Iteration & Objective & Iteration \\
\hline 1 & 15172 & 1022 & 15170.34 & 43 & 15170.34 & 43 & 15172 & 75 \\
\hline 2 & 13104.08 & 1242 & 13102.31 & 43 & 13102.31 & 43 & 13104.08 & 73 \\
\hline 3 & 13395.2 & 1180 & 13393.64 & 43 & 13393.65 & 43 & 13395.2 & 59 \\
\hline 4 & 13141.71 & 1372 & 13139.67 & 43 & 13139.67 & 43 & 13141.71 & 66 \\
\hline 5 & 12754.94 & 1212 & 12753.82 & 41 & 12753.82 & 41 & 12754.94 & 70 \\
\hline 6 & 13422.99 & 1047 & 13421.89 & 41 & 13421.89 & 41 & 13422.99 & 66 \\
\hline 7 & 11782.99 & 793 & 11781.44 & 43 & 11781.44 & 43 & 11782.99 & 56 \\
\hline 8 & 15201.93 & 1007 & 15199.67 & 43 & 15199.67 & 43 & 15201.93 & 79 \\
\hline 9 & 12873.23 & 805 & 12871.22 & 43 & 12871.23 & 43 & 12873.24 & 68 \\
\hline 10 & 13230.66 & 913 & 13229.05 & 43 & 13229.05 & 43 & 13230.66 & 60 \\
\hline 11 & 11346.39 & 1055 & 11345.02 & 41 & 11345.02 & 41 & 11346.39 & 89 \\
\hline 12 & 13625.79 & 1259 & 13623.66 & 43 & 13623.66 & 43 & 13625.79 & 77 \\
\hline 13 & 12858.7 & 966 & 12857.22 & 43 & 12857.22 & 43 & 12858.71 & 55 \\
\hline 14 & 13592.4 & 1058 & 13590.74 & 43 & 13590.74 & 43 & 13592.4 & 64 \\
\hline 15 & 13971.45 & 925 & 13969.87 & 43 & 13969.87 & 43 & 13971.45 & 67 \\
\hline 16 & 13348.72 & 1479 & 13347.35 & 41 & 13347.35 & 43 & 13348.72 & 77 \\
\hline 17 & 13164.36 & 940 & 13163.07 & 41 & 13163.07 & 41 & 13164.36 & 73 \\
\hline 18 & 14129.75 & 984 & 14128.09 & 43 & 14128.09 & 43 & 14129.75 & 55 \\
\hline 19 & 13470.62 & 891 & 13468.43 & 43 & 13468.43 & 43 & 13470.62 & 68 \\
\hline 20 & 14493.33 & 856 & 14491.95 & 43 & 14491.95 & 43 & 14493.33 & 65 \\
\hline 21 & 13841.5 & 944 & 13839.65 & 43 & 13839.65 & 43 & 13841.5 & 66 \\
\hline 22 & 13701.3 & 1438 & 13699.17 & 43 & 13699.17 & 43 & 13701.3 & 54 \\
\hline 23 & 14182.93 & 908 & 14181.34 & 43 & 14181.34 & 43 & 14182.93 & 65 \\
\hline 24 & 13957.9 & 985 & 13955.42 & 43 & 13955.43 & 43 & 13957.9 & 74 \\
\hline 25 & 14221.12 & 1228 & 14219.6 & 43 & 14219.6 & 43 & 14221.12 & 65 \\
\hline
\end{tabular}


Computational results for LHSCPLP, category B problem $50 \times 50 \times 50$.

\begin{tabular}{|c|c|c|c|c|c|c|c|c|}
\hline \multirow{2}{*}{ S.No } & \multicolumn{2}{|c|}{ R1_LHS } & \multicolumn{2}{|c|}{ R2_LHS } & \multicolumn{2}{|c|}{ R3_LHS } & \multicolumn{2}{|c|}{ R4_LHS } \\
\hline & Objective & Iteration & Objective & Iteration & Objective & Iteration & Objective & Iteration \\
\hline 1 & 497.088 & 107 & 497.088 & 58 & 526.801 & 88 & 526.8437 & 282 \\
\hline 2 & 563.076 & 105 & 563.076 & 59 & 595.003 & 34 & 595.0317 & 92 \\
\hline 3 & 705.075 & 101 & 705.067 & 31 & 732.775 & 84 & 732.8163 & 330 \\
\hline 4 & 798.315 & 116 & 798.32 & 37 & 818.037 & 16 & 818.0726 & 12 \\
\hline 5 & 1030.828 & 107 & 1030.828 & 35 & 1062.68 & 117 & 1062.729 & 267 \\
\hline 6 & 746.658 & 101 & 746.658 & 47 & 855.058 & 13 & 855.1125 & 541 \\
\hline 7 & 894.089 & 125 & 894.089 & 60 & 930.367 & 23 & 930.4226 & 129 \\
\hline 8 & 548.966 & 112 & 548.966 & 61 & 577.275 & 36 & 577.3666 & 74 \\
\hline 9 & 801.892 & 106 & 801.892 & 38 & 816.326 & 57 & 816.3609 & 45 \\
\hline 10 & 661.608 & 108 & 661.61 & 42 & 672.059 & 52 & 672.1011 & 13 \\
\hline 11 & 797.476 & 127 & 797.476 & 60 & 839.853 & 96 & 839.9097 & 316 \\
\hline 12 & 986.781 & 110 & 986.781 & 59 & 998.737 & 39 & 999.0034 & 141 \\
\hline 13 & 961.018 & 115 & 961.018 & 40 & 971.421 & 17 & 971.4302 & 75 \\
\hline 14 & 705.189 & 118 & 705.187 & 29 & 715.545 & 157 & 715.6213 & 246 \\
\hline 15 & 1044.981 & 118 & 1044.981 & 6 & 1064 & 4 & 1068.014 & 1488 \\
\hline 16 & 775.113 & 104 & 775.1 & 31 & 827.737 & 18 & 827.8167 & 130 \\
\hline 17 & 513.108 & 109 & 513.108 & 24 & 514.347 & 29 & 514.3696 & 12 \\
\hline 18 & 1148.365 & 91 & 1148.365 & 37 & 1168.512 & 53 & 1168.53 & 166 \\
\hline 19 & 516.55 & 117 & 516.56 & 129 & 573.696 & 28 & 573.7134 & 122 \\
\hline 20 & 808.334 & 108 & 808.334 & 117 & 839.348 & 60 & 839.4315 & 139 \\
\hline 21 & 684.192 & 115 & 684.192 & 41 & 701.988 & 72 & 702.016 & 154 \\
\hline 22 & 891.605 & 111 & 891.6 & 76 & 931.094 & 111 & 931.7247 & 282 \\
\hline 23 & 731.887 & 112 & 731.887 & 44 & 784.454 & 28 & 784.5266 & 164 \\
\hline 24 & 808.642 & 129 & 808.642 & 51 & 819.314 & 67 & 819.3718 & 47 \\
\hline 25 & 652.084 & 115 & 652.08 & 23 & 684.047 & 24 & 684.0895 & 79 \\
\hline
\end{tabular}


Computational results for RHSCPLP, category B problem $50 \times 50 \times 50$.

\begin{tabular}{|c|c|c|c|c|c|c|c|c|}
\hline \multirow{2}{*}{ S.No } & \multicolumn{2}{|c|}{ R1_RHS } & \multicolumn{2}{|c|}{ R2_RHS } & \multicolumn{2}{|c|}{ R3_RHS } & \multicolumn{2}{|c|}{ R4_RHS } \\
\hline & Objective & Iteration & Objective & Iteration & Objective & Iteration & Objective & Iteration \\
\hline 1 & 509.67 & 2380 & 509.67 & 42 & 538.511 & 62 & 538.5373 & 1062 \\
\hline 2 & 574.659 & 2468 & 574.65 & 74 & 606.901 & 73 & 607.0102 & 1611 \\
\hline 3 & 719.101 & 1867 & 719.105 & 70 & 746.441 & 95 & 746.4609 & 1191 \\
\hline 4 & 810.901 & 2722 & 810.86 & 81 & 830.328 & 70 & 830.362 & 17 \\
\hline 5 & 1042.539 & 2456 & 1042.539 & 70 & 1074.908 & 50 & 1074.91 & 2037 \\
\hline 6 & 755.642 & 2859 & 755.653 & 86 & 865.948 & 90 & 866.1968 & 5019 \\
\hline 7 & 906.407 & 2610 & 906.4 & 83 & 942.529 & 46 & 942.5336 & 599 \\
\hline 8 & 558.779 & 3002 & 558.779 & 81 & 585.691 & 77 & 585.7031 & 188 \\
\hline 9 & 820.528 & 2086 & 820.5 & 76 & 833.97 & 67 & 833.9829 & 190 \\
\hline 10 & 673.108 & 2475 & 673.076 & 70 & 683.48 & 54 & 683.486 & 206 \\
\hline 11 & 805.905 & 2799 & 805.9 & 91 & 848.007 & 77 & 848.0675 & 892 \\
\hline 12 & 993.454 & 2457 & 993.44 & 90 & 1005.197 & 84 & 1005.206 & 2672 \\
\hline 13 & 974.165 & 2377 & 974.16 & 83 & 983.737 & 73 & 983.8542 & 145 \\
\hline 14 & 718.786 & 1854 & 718.7 & 48 & 728.797 & 95 & 728.8193 & 2267 \\
\hline 15 & 1057.976 & 2766 & 1057.96 & 83 & 1080.574 & 53 & 1080.589 & 3897 \\
\hline 16 & 786.446 & 3070 & 786.44 & 81 & 838.286 & 49 & 838.2869 & 906 \\
\hline 17 & 523.433 & 2614 & 523.45 & 80 & 524.643 & 61 & 524.6626 & 22 \\
\hline 18 & 1157.567 & 2758 & 1157.567 & 83 & 1177.199 & 58 & 1177.203 & 1171 \\
\hline 19 & 532.823 & 2395 & 532.77 & 78 & 587.822 & 69 & 587.8443 & 433 \\
\hline 20 & 821.588 & 2436 & 821.57 & 69 & 854.055 & 58 & 854.0678 & 501 \\
\hline 21 & 696.342 & 2614 & 696.342 & 76 & 713.355 & 62 & 713.3634 & 1328 \\
\hline 22 & 899.603 & 2410 & 899.5 & 67 & 939.821 & 91 & 939.8355 & 124 \\
\hline 23 & 744.329 & 2348 & 744.319 & 88 & 795.562 & 58 & 795.5632 & 2627 \\
\hline 24 & 815.427 & 3415 & 815.422 & 89 & 827.091 & 50 & 827.1029 & 370 \\
\hline 25 & 669.165 & 2064 & 669.16 & 94 & 700.632 & 63 & 700.6466 & 182 \\
\hline
\end{tabular}




\section{Appendix B}

Computational results for category $\mathbf{P 1}$ problems $50 \times 50 \times 50$. (time in seconds).

\begin{tabular}{|c|c|c|c|c|c|c|c|c|c|}
\hline \multirow{2}{*}{ S.No } & \multirow{2}{*}{ Optimal } & \multicolumn{2}{|c|}{ BB } & \multicolumn{2}{|c|}{$\mathrm{R} 1$} & \multicolumn{2}{|c|}{$\mathrm{R} 2$} & \multicolumn{2}{|c|}{ R3 } \\
\hline & & Nodes & Time & Nodes & Time & Nodes & Time & Nodes & Time \\
\hline 1 & 3628.5500 & 319 & 5260 & 293 & 4786 & 269 & 4513 & 269 & 4516 \\
\hline 2 & 4177.1810 & 137 & 1741 & 137 & 1666 & 125 & 1500 & 125 & 1525 \\
\hline 3 & 3867.7100 & 65 & 909 & 61 & 789 & 57 & 711 & 49 & 706 \\
\hline 4 & 5747.2630 & 117 & 1600 & 111 & 1525 & 111 & 1431 & 95 & 1339 \\
\hline 5 & 4077.6970 & 187 & 3163 & 175 & 2971 & 175 & 2873 & 175 & 2905 \\
\hline 6 & 3541.6310 & 69 & 888 & 65 & 811 & 43 & 467 & 43 & 457 \\
\hline 7 & 5407.8320 & 109 & 1571 & 93 & 1297 & 93 & 1248 & 93 & 1436 \\
\hline 8 & 5015.8550 & 57 & 662 & 45 & 428 & 45 & 426 & 31 & 382 \\
\hline 9 & 4271.4190 & 121 & 1707 & 119 & 1598 & 119 & 1597 & 91 & 1374 \\
\hline 10 & 3725.4900 & 131 & 2016 & 119 & 1747 & 119 & 1743 & 119 & 1727 \\
\hline 11 & 4856.1350 & 211 & 2105 & 201 & 1920 & 163 & 1536 & 139 & 1372 \\
\hline 12 & 3553.6670 & 615 & 7072 & 519 & 5883 & 499 & 5645 & 447 & 5240 \\
\hline 13 & 4115.5330 & 65 & 703 & 45 & 416 & 45 & 409 & 45 & 406 \\
\hline 14 & 5218.9520 & 13 & 261 & 13 & 272 & 13 & 264 & 13 & 240 \\
\hline 15 & 5413.4630 & 297 & 3428 & 283 & 3179 & 271 & 3030 & 259 & 2880 \\
\hline 16 & 4952.1770 & 167 & 2192 & 145 & 1822 & 145 & 1824 & 145 & 1830 \\
\hline 17 & 4593.6770 & 483 & 5339 & 359 & 3879 & 359 & 3885 & 355 & 3837 \\
\hline 18 & 4983.7330 & 297 & 4970 & 285 & 4676 & 235 & 3844 & 235 & 3848 \\
\hline 19 & 4566.5570 & 364 & 4893 & 361 & 4759 & 243 & 3182 & 243 & 3202 \\
\hline 20 & 4783.2700 & 401 & 4586 & 329 & 3682 & 271 & 3009 & 271 & 3019 \\
\hline
\end{tabular}


Computational results for category P2 problems $50 \times 50 \times 50$.

\begin{tabular}{|c|c|c|c|c|c|c|c|c|c|}
\hline \multirow{2}{*}{ S.No } & \multirow{2}{*}{ Optimal } & \multicolumn{2}{|c|}{ BB } & \multicolumn{2}{|c|}{$\mathrm{R} 1$} & \multicolumn{2}{|c|}{ R2 } & \multicolumn{2}{|c|}{ R3 } \\
\hline & & Nodes & Time & Nodes & Time & Nodes & Time & Nodes & Time \\
\hline 1 & 832.7146 & 33 & 748 & 33 & 742 & 33 & 736 & 33 & 735 \\
\hline 2 & 656.6308 & 99 & 1844 & 89 & 1618 & 89 & 1622 & 89 & 1644 \\
\hline 3 & 375.7824 & 17 & 376 & 13 & 264 & 13 & 264 & 13 & 260 \\
\hline 4 & 547.0326 & 19 & 646 & 19 & 631 & 19 & 607 & 19 & 599 \\
\hline 5 & 991.5337 & 57 & 1297 & 57 & 1254 & 57 & 1279 & 57 & 1254 \\
\hline 6 & 548.5699 & 65 & 1594 & 65 & 1578 & 65 & 1555 & 65 & 1572 \\
\hline 7 & 561.4253 & 23 & 673 & 23 & 675 & 23 & 641 & 23 & 628 \\
\hline 8 & 670.8930 & 75 & 1427 & 67 & 1251 & 67 & 1259 & 67 & 1259 \\
\hline 9 & 547.2954 & 51 & 1165 & 51 & 1155 & 51 & 1132 & 51 & 1147 \\
\hline 10 & 632.5004 & 11 & 215 & 7 & 118 & 7 & 98 & 7 & 97 \\
\hline 11 & 473.5373 & 11 & 219 & 9 & 132 & 9 & 134 & 9 & 129 \\
\hline 12 & 566.0262 & 11 & 227 & 11 & 219 & 9 & 132 & 9 & 133 \\
\hline 13 & 583.2800 & 35 & 577 & 35 & 575 & 35 & 574 & 35 & 546 \\
\hline 14 & 521.6505 & 53 & 1129 & 53 & 1110 & 53 & 1102 & 53 & 1114 \\
\hline 15 & 702.6561 & 15 & 310 & 15 & 326 & 15 & 328 & 15 & 270 \\
\hline 16 & 573.2853 & 13 & 333 & 11 & 266 & 11 & 264 & 11 & 241 \\
\hline 17 & 940.2433 & 119 & 2975 & 119 & 2972 & 119 & 2968 & 119 & 2919 \\
\hline 18 & 572.7860 & 37 & 707 & 37 & 687 & 37 & 688 & 37 & 679 \\
\hline 19 & 493.0821 & 27 & 855 & 25 & 752 & 25 & 747 & 25 & 743 \\
\hline 20 & 558.8294 & 57 & 1421 & 57 & 1415 & 53 & 1284 & 53 & 1306 \\
\hline
\end{tabular}


Computational results for category P3 problems $100 \times 100 \times 100$.

\begin{tabular}{|c|c|c|c|c|c|c|c|c|c|}
\hline \multirow{2}{*}{ S.No } & \multirow{2}{*}{ Optimal } & \multicolumn{2}{|c|}{ BB } & \multicolumn{2}{|c|}{$\mathrm{R} 1$} & \multicolumn{2}{|c|}{ R2 } & \multicolumn{2}{|c|}{ R3 } \\
\hline & & Nodes & Time & Nodes & Time & Nodes & Time & Nodes & Time \\
\hline 1 & 37229.89 & 1217 & 210810 & 1217 & 210780 & 1213 & 207760 & 1013 & 175297.9 \\
\hline 2 & 40632.64 & 219 & 43232 & 203 & 39138 & 203 & 39114 & 171 & 33650.49 \\
\hline 3 & 45733.24 & 383 & 77366 & 363 & 73386 & 346 & 69842 & 316 & 63670 \\
\hline 4 & 44268.57 & 1539 & 283600 & 1517 & 279643 & 1503 & 276771 & 1399 & 257673.4 \\
\hline 5 & 39611.71 & 878 & 170332 & 796 & 154451 & 760 & 147288 & 612 & 118623 \\
\hline 6 & 40424.14 & 405 & 97605 & 390 & 94082 & 328 & 78889 & 328 & 78877 \\
\hline 7 & 38088.52 & 571 & 121623 & 484 & 103126 & 465 & 98901 & 460 & 97881 \\
\hline 8 & 42114.38 & 308 & 53284 & 305 & 52823 & 283 & 48770 & 268 & 46284 \\
\hline 9 & 40042.96 & 631 & 163429 & 625 & 161924 & 612 & 158394 & 483 & 124922 \\
\hline 10 & 40399.63 & 624 & 154128 & 590 & 145761 & 547 & 135022 & 531 & 131001 \\
\hline 11 & 35108.18 & 935 & 201960 & 872 & 188436 & 801 & 172838 & 687 & 148314 \\
\hline 12 & 41030.85 & 2571 & 732735 & 2151 & 613087 & 2124 & 605149 & 1915 & 545664 \\
\hline 13 & 37272.51 & 342 & 77292 & 284 & 64212 & 241 & 54319 & 241 & 54354 \\
\hline 14 & 41362.47 & 196 & 41944 & 188 & 40331 & 160 & 34163 & 160 & 34137 \\
\hline 15 & 38363.8 & 1315 & 318230 & 1275 & 308621 & 1162 & 281116 & 1151 & 278457 \\
\hline 16 & 37185.86 & 761 & 196338 & 710 & 183218 & 568 & 146358 & 568 & 146448 \\
\hline 17 & 40360.54 & 2062 & 583546 & 1566 & 443250 & 1511 & 427479 & 1477 & 417826 \\
\hline 18 & 37376.5 & 1275 & 336600 & 1209 & 319200 & 1005 & 265121 & 1005 & 265264 \\
\hline 19 & 42302.74 & 1617 & 350889 & 1561 & 338820 & 1257 & 272619 & 1251 & 271292 \\
\hline 20 & 39393.8 & 1714 & 368510 & 1394 & 299780 & 1260 & 270724 & 1260 & 270701 \\
\hline
\end{tabular}


Computational results for category P4 problems $100 \times 100 \times 100$.

\begin{tabular}{|c|c|c|c|c|c|c|c|c|c|}
\hline \multirow{2}{*}{ S.No } & \multirow{2}{*}{ Optimal } & \multicolumn{2}{|c|}{ BB } & \multicolumn{2}{|c|}{$\mathrm{R} 1$} & \multicolumn{2}{|c|}{ R2 } & \multicolumn{2}{|c|}{ R3 } \\
\hline & & Nodes & Time & Nodes & Time & Nodes & Time & Nodes & Time \\
\hline 1 & 8709.583 & 425 & 106250 & 425 & 106112 & 373 & 93216 & 373 & 93210 \\
\hline 2 & 9476.864 & 25 & 3995.1 & 25 & 3854.1 & 13 & 2026.452 & 13 & 1985.452 \\
\hline 3 & 9674.657 & 71 & 15926 & 71 & 15823 & 71 & 15888 & 69 & 15413.38 \\
\hline 4 & 10010.77 & 995 & 267790 & 995 & 267725 & 995 & 267693 & 932 & 250745.5 \\
\hline 5 & 8624.385 & 189 & 44226 & 189 & 44117 & 142 & 33195 & 142 & 33151 \\
\hline 6 & 9308.648 & 414 & 102672 & 414 & 102642 & 414 & 102618 & 384 & 95187 \\
\hline 7 & 8012.688 & 370 & 85840 & 370 & 85794 & 274 & 63525 & 274 & 63526 \\
\hline 8 & 9758.747 & 988 & 211432 & 883 & 188830 & 848 & 181444 & 786 & 168167 \\
\hline 9 & 9351.761 & 587 & 102725 & 587 & 102666 & 440 & 76910 & 440 & 76904 \\
\hline 10 & 9436.933 & 1080 & 190080 & 1067 & 187697 & 1067 & 187754 & 986 & 173438 \\
\hline 11 & 9331.35 & 119 & 28203 & 97 & 22909 & 97 & 22935 & 97 & 22942 \\
\hline 12 & 9282.44 & 1181 & 232657 & 1181 & 232619 & 934 & 183966 & 934 & 183930 \\
\hline 13 & 7862.446 & 601 & 109983 & 601 & 109938 & 590 & 107879 & 532 & 97257 \\
\hline 14 & 9388.992 & 547 & 126904 & 547 & 126768 & 547 & 126812 & 498 & 115482 \\
\hline 15 & 9494.626 & 164 & 31488 & 164 & 31357 & 139 & 26636 & 139 & 26642 \\
\hline 16 & 9327.953 & 1144 & 257400 & 968 & 217726 & 824 & 185346 & 824 & 185317 \\
\hline 17 & 9417.17 & 1326 & 290394 & 1316 & 288068 & 1296 & 283760 & 1296 & 283744 \\
\hline 18 & 8126.866 & 488 & 89792 & 481 & 88445 & 358 & 65822 & 358 & 65805 \\
\hline 19 & 8095.194 & 1402 & 291616 & 1298 & 269873 & 1298 & 269934 & 1285 & 267208 \\
\hline 20 & 9329.764 & 1205 & 256665 & 1202 & 255878 & 1094 & 232970 & 1094 & 232993 \\
\hline
\end{tabular}

